\title{
Analysis of Ulysses data: Radiation pressure effects on dust particles
}

\author{
A. Wehry ${ }^{1}$, H. Krüger ${ }^{2}$, and E. Grün ${ }^{2,3}$ \\ 1 SAP AG, Neurottstrasse 16, 69190 Walldorf, Germany \\ 2 Max-Planck-Institut für Kernphysik, Saupfercheckweg 1, 69117 Heidelberg, Germany \\ ${ }^{3}$ Hawaii Institute of Geophysics and Planetology, HIGP, University of Hawaii, 1680 East West Road, Honolulu, \\ HI 96822, USA
}

Received 3 November 2003 / Accepted 18 February 2004

\begin{abstract}
The objective of this paper is to illustrate the influence of the radiation pressure and the electromagnetic force on dust particles in interplanetary space. Between 1990 and 200162 so-called $\beta$-meteoroids $(\beta$ describes the ratio of the radiation pressure force to gravity) were detected as dust particles coming from the inner solar system on hyperbolic orbits. 24 of them were detected shortly after the launch of Ulysses within the ecliptic and 38 of them were recorded primarily passing the Solar poles. Furthermore high speed particles not coming from the direction of the Sun were ejected from the solar system by electromagnetic forces. For the time period until the end of 2008 the effective area for particles coming from the direction of the Sun has been determined. The particles' perihelion distances indicated that they originated within a region of 0.5 AU from the Sun. For the second orbit of Ulysses the flux of $\beta$-meteoroids was determined using the same method as during its first revolution. Because of the more defocusing phase during the first orbit of Ulysses the production rate of $\beta$-meteoroids has been estimated to be nearly twice of that for the second revolution. On the other hand, an imbalance of the identified $\beta$-meteoroids between the north and south pole could not be explained by the solar cycle.
\end{abstract}

Key words. meteors, meteroids - solar system: general - ISM: dust, extinction

\section{Introduction}

After the Ulysses spacecraft was launched in October 1990 the flyby of Jupiter caused the spacecraft to move into an orbit which is nearly perpendicular to the ecliptic plane. The first flight through perihelion occurred on March 1995 at a solar distance of 1.3 AU, whereas the second one happened in May 2002. On board the spin-stabilized Ulysses spacecraft there is the Ulysses dust detector with a sensor area of $0.1 \mathrm{~m}^{2}$. This detector, described in detail in Grün et al. (1992a,b), is a multicoincidence impact ionization detector with its spin axis pointing towards the Earth. The detection geometry of this detector is determined by a viewing cone of $140^{\circ}$ relative to the sensor axis. The sensor axis is mounted at $85^{\circ}$ to the spin axis. The measured particles are within a mass range of $10^{-18}$ to $10^{-8} \mathrm{~kg}$ with impact speeds from 2 to $70 \mathrm{~km} \mathrm{~s}^{-1}$ (Grün et al. 1992b). The uncertainty of the mass determination amounts to a factor of 10 whereas the uncertainty of the speed is a factor of 2 .

Based on measurements on board the Pioneer 8 and 9 spacecraft, dust particles in hyperbolic orbits moving away from the solar direction have been identified by Berg \& Grün (1973) and by Zook \& Berg (1975) for the first time. These

Send offprint requests to: A. Wehry, e-mail: andreas.wehry@sap.com particles are called $\beta$-meteoroids and are escaping from the Solar system in unbound orbits when the repelling force from the solar radiation becomes similar in strength to the solar gravity. The detection of $\beta$-meteoroids from the data set of the Ulysses dust experiment has been investigated by Wehry \& Mann (1999). In the data set from Krüger et al. $(1999,2001)$, we identified $\beta$-meteoroids from the remaining interplanetary particles after subtracting Jovian dust streams and interstellar dust particles. $\beta$-meteoroids are formed whenever small particles are generated in interplanetary space. This can occur through sublimation within 20 solar radii of the Sun or through collisions as a result of fragmentation of bigger particles. If these fragments are small enough they will escape from the gravitation field of the Sun on hyperbolic orbits. Using these assumptions concerning the origin of $\beta$-meteoroids as well as the detector sensitivity we identified $\beta$-meteoroids from the interplanetary dust component in accordance with the criterion that was developed in Wehry \& Mann (1999). During the first 100 days of the Ulysses mission as well as during the south and north polar passage $46 \beta$-meteoroids were detected with perihelion distances smaller than 0.5 AU. Apart from these $\beta$-meteoroids coming from the direction of the Sun the influence of the radiation pressure force also causes $\beta$-meteoroids to come from a region further than 0.5 AU from the Sun. 


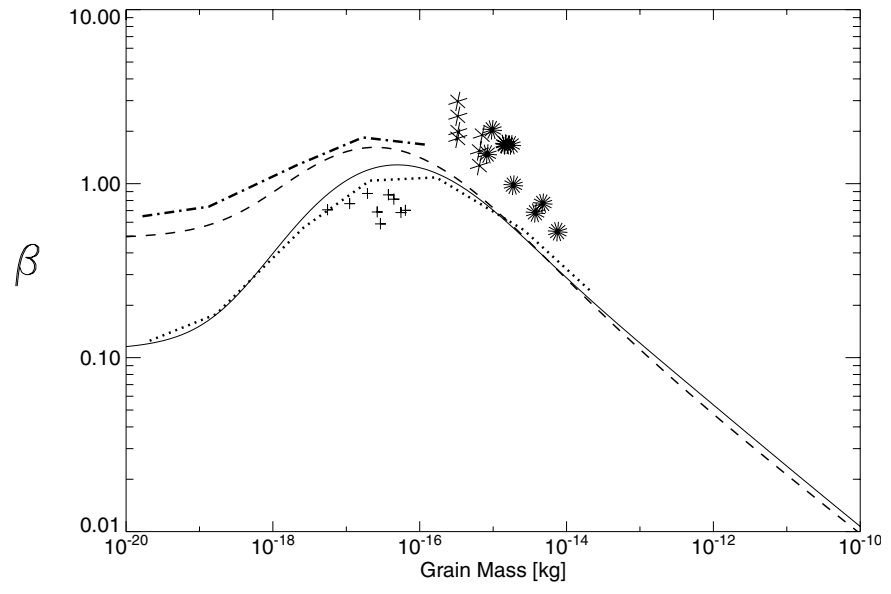

Fig. 1. Radiation pressure coefficient $\beta$ as a function of the particle mass: - : homogenous astronomical silicate $\left(2.5 \times 10^{3} \mathrm{~kg} / \mathrm{m}^{3}\right) ; \cdots$ : porous astronomical silicate averaged over different orientations; - - -: spherical porous material $\left(2.5 \times 10^{3} \mathrm{~kg} / \mathrm{m}^{3}\right) ;-\cdot-$ : particles composed of organic material; +: compact aggregates of astronomical silicate; $*$ : porous aggregates of astronomical silicate; $\star$ : core-mantle-particles of organical material (cf. Landgraf et al. 1999).

In this case the influence of the electromagnetic force cannot be neglected.

In the current paper we will concentrate on the following time periods: for the time up to the end of 2001 we will show how many $\beta$-meteoroids have been identified using the above-mentioned criterion. We will investigate the connection between the occurrence of $\beta$-meteoroids and the solar magnetic field. Using the number of identified $\beta$-meteoroids during the first orbit of Ulysses we will give an estimate for the flux of $\beta$-meteoroids for this time period. Furthermore, we will determine the effective area of the detector until the end of 2008 in order to present the time span in which $\beta$-meteoroids can be identified.

\section{Dynamics of the interplanetary dust}

Because of the solar gravitational attraction force $\boldsymbol{F}_{\mathrm{g}}$, interplanetary particles move on Keplerian orbits around the Sun. Additionally, the dynamics of small particles is influenced by the radiation pressure force $\boldsymbol{F}_{\mathrm{r}}=-\beta \cdot \boldsymbol{F}_{\mathrm{g}}$ which is in the opposite direction to $\boldsymbol{F}_{\mathrm{g}}$. The so-called $\beta$-value or the radiation pressure coefficient, shown in Fig. 1, describes the ratio of the radiation pressure $\boldsymbol{F}_{\mathrm{r}}$ to gravity $\boldsymbol{F}_{\mathrm{g}} . \boldsymbol{F}_{\mathrm{r}}$ counteracts the solar attraction and leads to an effective force $\boldsymbol{F}_{\text {eff }}=(1-\beta) \cdot \boldsymbol{F}_{\mathrm{g}}$ describing this reduced gravitation field.

From the orbital energy we obtain the escape velocity $v_{\mathrm{esc}}=$ $\sqrt{2 \mu\left(\frac{1}{r}-\frac{1}{2 a}\right)}$ is needed for a particle to leave the bound orbit ( $\mu$ is the reduced mass, $r$ the solar distance and $a$ the semimajor axis). Taking the radiation pressure force into account, the escape velocity $v_{\text {esc }}$ decreases with increasing $\beta$ proportional to $\sqrt{1-\beta}$ compared to the case when only gravity applies. The value of $\beta$, shown in Fig. 1, is independent of the solar distance $r$ and depends only on the physical properties, e.g. on the mass, material, composition and structure, as well as on the size of the dust particle. Particles within the mass range of $10^{-18}$
Table 1. The gravitational force $F_{\mathrm{g}}$ and the Lorentz force $F_{\mathrm{L}}$ acting on a dust particle with a radius $a$ have been compared under typical interplanetary conditions (at $1 \mathrm{AU}$, solar wind with $v_{\mathrm{sw}}=400 \mathrm{~km} \mathrm{~s}^{-1}$, magnetic field $B=5 n T$, particles charged to $U=10 \mathrm{~V}$; forces are given in Newton).

\begin{tabular}{cccc}
\hline \hline$a(\mu \mathrm{m})$ & $F_{\mathrm{g}}(\mathrm{N})$ & $F_{\mathrm{L}}(\mathrm{N})$ & $\frac{F_{\mathrm{L}}}{F_{\mathrm{g}}}$ \\
\hline 0.1 & $8.7 \times 10^{-20}$ & $1.6 \times 10^{-19}$ & 1.84 \\
1 & $8.7 \times 10^{-17}$ & $1.6 \times 10^{-18}$ & $1.84 \times 10^{-2}$ \\
10 & $8.7 \times 10^{-14}$ & $1.6 \times 10^{-17}$ & $1.84 \times 10^{-4}$ \\
100 & $8.7 \times 10^{-12}$ & $1.6 \times 10^{-16}$ & $1.84 \times 10^{-5}$ \\
\hline
\end{tabular}

to $10^{-16} \mathrm{~kg}$ have $\beta$-values of from 0.3 to 1.7 and are the source of $\beta$-meteoroids.

For dust particles the effective gravitational force decreases with the solar distance proportional to $r^{-2}$, whereas the azimuthal magnetic field and the Lorentz force $\boldsymbol{F}_{\mathrm{L}}=\frac{Q}{c}\left(\boldsymbol{v}_{\text {rel }} \times \boldsymbol{B}\right)$ ( $Q$ : charge, $c$ : speed of light, $\boldsymbol{v}_{\text {rel }}$ : relative velocity between particle $\boldsymbol{v}$ and solar wind $\boldsymbol{v}_{\mathrm{sw}}, \boldsymbol{B}$ : magnetic field) decreases proportional to $r^{-1}$. The charge on a spherical particle is given by $Q=4 \pi \epsilon_{0} U a$ with permittivity $\epsilon_{0}=8.859 \times 10^{-12} \frac{\mathrm{C}}{\mathrm{Vm}}$, surface potential $U$ and grain radius $a$. The solar wind velocity, which amounts to nearly $400 \mathrm{~km} \mathrm{~s}^{-1}$, is radial and for the greater part of the particle orbits $v_{\mathrm{sw}} \gg v$, whereas the Lorentz force is primarily normal to the orbit. A comparison between the gravitational force $F_{\mathrm{g}}$ and the Lorentz force $F_{\mathrm{L}}$ acting on a dust particle under typical interplanetary conditions at $1 \mathrm{AU}$ is shown in Table 1. Small particles are mainly dominated by the magnetic force whereas bigger particles are dominated by the gravitational force. For dust particles with radii smaller than $0.2 \mu \mathrm{m}$ the influence of the Lorentz force beyond a solar distance of $2 \mathrm{AU}$ is greater than that of the gravitational force. So the Lorentz force is responsible for the occurrence of $\beta$-meteoroids at higher solar latitudes.

Because of an alternating magnetic polarity near the ecliptic plane and because of a single polarity at high ecliptic latitudes the average force on particles close to the ecliptic is much smaller than that on high-latitude particles. The flux of interstellar dust grains, which is continuously monitored by Ulysses, began to decrease in mid-1996. The observed decrease by a factor of 3 was interpreted to be the result of the electro-magnetic interaction of the grains with the solar wind magnetic field (Landgraf 2000). The polarity of the solar wind magnetic field changes with the 22-year solar cycle. During the solar maximum in 1991 the field polarity became northpointing. The azimuthal component of the corresponding radially expanding (Parker 1958) spiral field deflects interstellar grains in the northern hemisphere to the north and in the southern hemisphere to the south. Thus, the net effect of the interplanetary magnetic field during the 1991 polarity cycle was to divert interstellar dust out of the solar system. During the 2000/2001 solar maximum the field polarity reversed again. Then, interstellar grains in the northern hemisphere were deflected to the south and grains in the southern hemisphere to the north. This focusing effect increased the flux of interstellar grains in the inner Solar System significantly, starting in 2002 
Table 2. Model calculations to find the minimum speed and maximum deviation of the ejected particle from the solar direction. The parent objects are moving on circular orbits (cf. Wehry \& Mann 1999).

\begin{tabular}{ccccc}
\hline \hline$e_{0}$ & $q(\mathrm{AU})$ & $\beta$ & $\begin{array}{c}\gamma\left({ }^{\circ}\right) \\
2.5 \mathrm{AU}\end{array}$ & $\begin{array}{c}v\left(\mathrm{~km} \mathrm{~s}^{-1}\right) \\
2.5 \mathrm{AU}\end{array}$ \\
\hline 0 & 0.3 & 0.5 & 14 & 19 \\
& 0.8 & 0.5 & 24 & 19 \\
0.3 & 0.3 & 0.4 & 11 & 27 \\
& 0.8 & 0.4 & 21 & 27 \\
0.6 & 0.3 & 0.2 & 14 & 24 \\
& 0.8 & 0.2 & 24 & 24 \\
0.9 & 0.3 & 0.2 & 9 & 38 \\
& 0.8 & 0.2 & 19 & 38 \\
\hline
\end{tabular}

$e_{0}$ eccentricity of the parent body;

$q$ perihelion distance;

$\gamma$ angle between the direction of the Sun and the particle velocity;

$v$ heliocentric speed.

(Landgraf et al. 2003). This means that positively charged particles will be carried out of the ecliptic primarily during the defocusing time period from 1991 to 2002 whereas during the focusing time period (from 2002 to 2013) they will be focussed to the ecliptic (Hamilton et al. 1996).

\section{Identification of $\beta$-meteoroids}

Micron-sized particles are assumed to move in Keplerian orbits around the Sun. Furthermore, we assume that $\beta$-meteoroids are produced from bigger particles at the perihelion of their orbit. The perihelion velocity of the fragment is assumed to be the same as the velocity of its parent body. For different perihelion distances within $1 \mathrm{AU}$ from the Sun and for different $\beta$-values the speed of the newly-formed $\beta$-meteoroid and the deviation from the direction of the Sun have been determined. Table 2 gives an example of the model calculation for the eccentricity and the perihelion distance of the orbit of the parent body and the assumed $\beta$-value of the fragment. This $\beta$-value is the lowest possible value for which the new particle can be in an unbound orbit. For example, assuming a circular orbit of the parent body at $0.3 \mathrm{AU}$ and a $\beta$-value of 0.5 the heliocentric speed of the new particle is $19 \mathrm{~km} \mathrm{~s}^{-1}$ at $2.5 \mathrm{AU}$ and the direction differs by $14^{\circ}$ from the radial direction. Comparing this $14^{\circ}$ to the detection cone of the experiment, which amounts to $140^{\circ}$, such a flux of particles can be assumed as directed radially outward. The same is the case for particles with higher $\beta$-values or for particles with higher perihelion velocities, when the orbits of the parent bodies are not longer circular. Depending on the solar distance we determined a minimum speed of 20 to $30 \mathrm{~km} \mathrm{~s}^{-1}$ as well as a maximum deviation of $20^{\circ}$ from the direction of the Sun (cf. Fig. 2).

To know the time period over which particles with different speeds can be seen from the direction of the Sun we determined the relative velocity to the spacecraft as well as the angle between the relative velocity to the spacecraft and the spin axis. This allows us to calculate the effective area from the periods of time during which $\beta$-meteoroids can be identified.

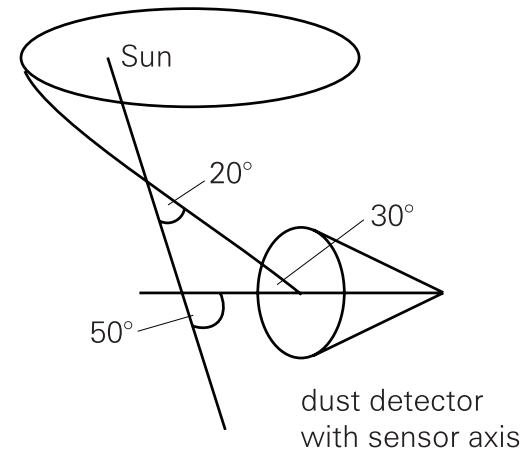

Fig. 2. Detection geometry for the identification of $\beta$-meteoroids: particles can be identified as $\beta$-meteoroids, if the angle between the Sun and the sensor axis of the detector at the time of the impact event is less than about $50^{\circ}$. This value results on the one hand from the model calculation $\left(20^{\circ}\right)$ mentioned in the text and on the other hand from the geometry of the detector $\left(30^{\circ}\right)$ (cf. Wehry \& Mann 1999).

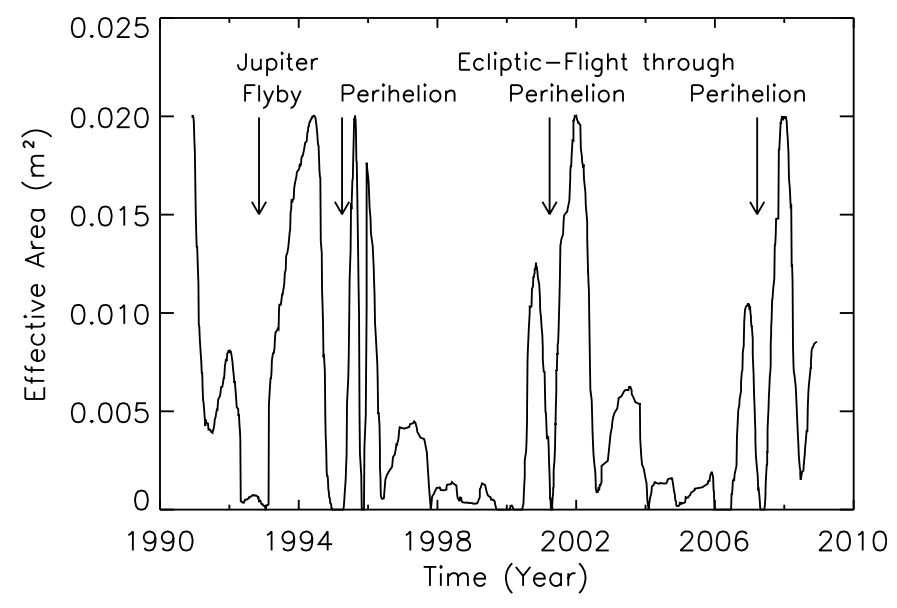

Fig. 3. The effective detection area of the dust experiment for particles incoming from the radial direction of the Sun is shown as a function of time until the end of 2008. This area is high in 1991 directly after launch and again shortly before and after the passage through the ecliptic plane during the perihelion of the Ulysses trajectory.

Figure 3 shows the effective area until the end of 2008. $\beta$-meteoroids can be seen at the beginning of the Ulyssesmission as well as shortly before and after the flyby through the perihelion of the orbit of Ulysses. The identification of $\beta$-meteoroids has been done with the help of an estimate using the model assumption mentioned above as well as the direction sensitivity of the dust detector. The angle of the sensor axis to the direction of the Sun must not exceed the value of $50^{\circ}$. This value results from the model calculation and from the detector geometry. On the other hand, we have a minimum particle speed of 20 to $30 \mathrm{~km} \mathrm{~s}^{-1}$. Using this method, 62 particles are identified as particles coming from the solar direction on hyperbolic orbits. 24 of them are detected within the ecliptic at a solar distances of from 1.0 to $1.6 \mathrm{AU}$ shortly after the launch. This result is in accordance with an earlier study of Baguhl (1993) who determined the same number of $\beta$-meteoroids during this time period using another method. 38 of these $\beta$ meteoroids were identified during the first passage of Ulysses over the Solar poles at a Solar distance of 1.8 to $2.7 \mathrm{AU}$. 


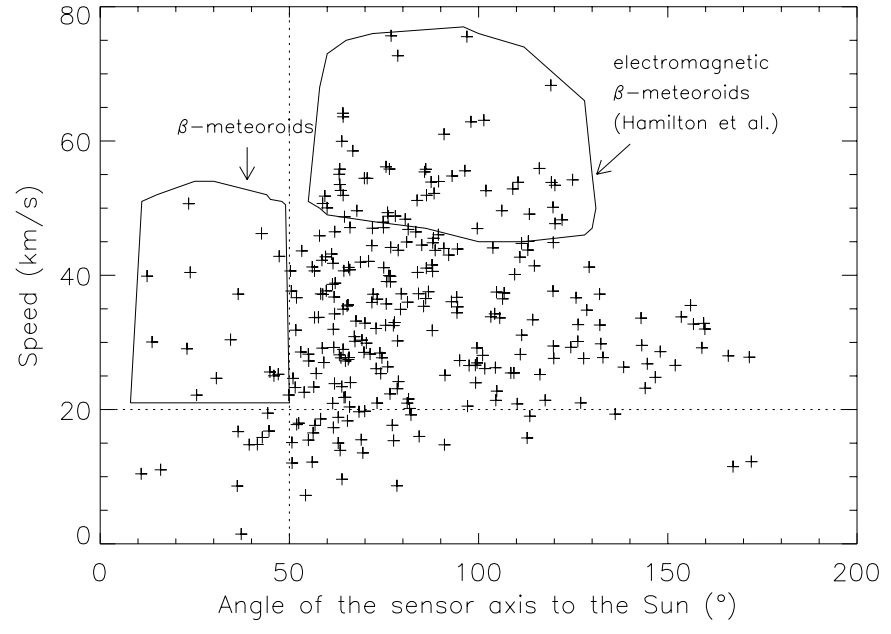

Fig. 4. The speed of the interplanetary particles vs. the angle of the sensor axis to the Sun provides the identification of $\beta$-meteoroids for the time period 16 August 1995-19 October 2001. $16 \beta$-meteoroids coming from the direction of the Sun are shown in the upper left quadrant whereas electromagnetical $\beta$-meteoroids according to Hamilton et al. (1996) are presented in the upper right quadrant.

The detection above the Solar poles is possible because of the influence of the Lorentz force $\boldsymbol{F}_{\mathrm{L}}$ on the dust particles (Hamilton et al. 1996).

In particular, the numerical imbalance of the identified $\beta$ meteoroids between the northern and southern hemisphere has been conspicuous. During the first revolution of Ulysses around the Sun $22 \beta$-meteoroids are identified, 18 of them within the northern hemisphere and 4 of them within the southern hemisphere. During the second revolution 16 particles are detected as $\beta$-meteoroids, all of them within the northern hemisphere.

For the time period of 16 August 1995-19 October 2001 Fig. 4 shows the speed of the interplanetary particles vs. the angle of the sensor axis to the Sun. The dotted lines characterize the criterion used to identify the $16 \beta$-meteoroids; they are shown in the upper left quadrant. Furthermore, in the upper right quadrant in Fig. 4 there are many of particles with a speed higher than $40 \mathrm{~km} \mathrm{~s}^{-1}$. These particles are escaping from the ecliptic region on unbound orbits.

To obtain information concerning the place and mechanism of their origin we determined the perihelion distance of the identified $\beta$-meteoroids. Taking the velocity and the position of the spacecraft into account, the resulting distribution for the average perihelion distances, including the uncertainty in the speed determination, is shown in Fig. 5. The perihelion distances determined $\leq 0.5$ AU (cf. Wehry \& Mann 1999). We concluded that the place of origin of $\beta$-meteoroids is within a region of $0.5 \mathrm{AU}$ from the Sun. Because sublimation only occurs at distances of up to 20 solar radii, these particles are probably formed through collisions.

\section{Discussion}

In a limited distance range from 1.3 AU to 2.3 AU the Ulysses spacecraft passed from close to the solar south pole through the ecliptic plane to close to the solar north pole. During 360 days

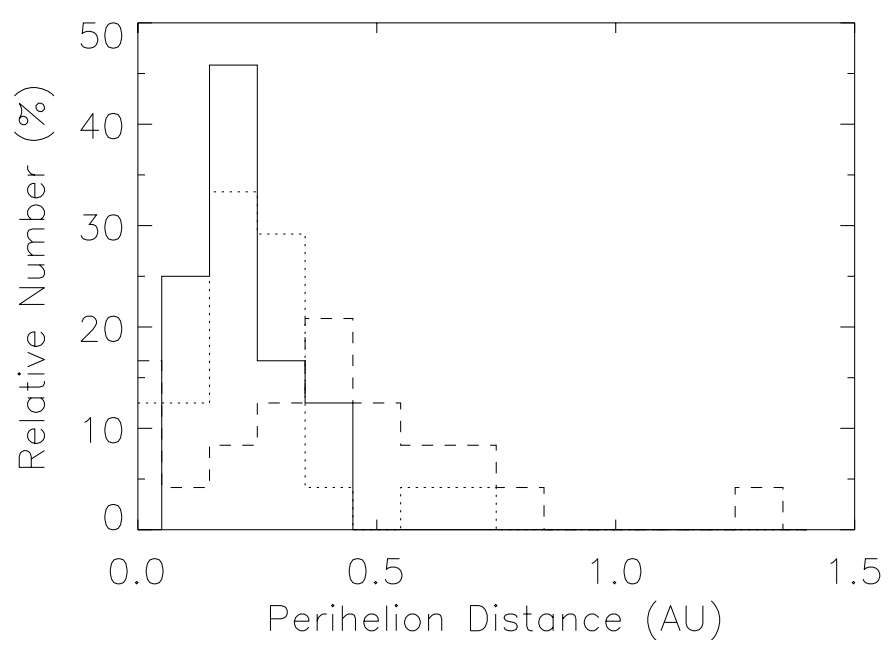

Fig. 5. The distribution of the perihelion distances of $\beta$-meteoroids for the out-of-ecliptic part is given, including the uncertainty in the speed determination (cf. Wehry \& Mann 1999).

of the first pole-to-pole passage an rate of 0.33 impacts per day or a total of 117 impacts of interplanetary dust particles was detected (Grün et al. 1997, 2001). Compared to this, during the first revolution of Ulysses only $22 \beta$-meteoroids were detected within a time period of 130 days which corresponds to an impact rate of 0.16 impacts per day. For the second revolution of Ulysses only $16 \beta$-meteoroids within a time period of 118 days correspond to an impact rate of 0.14 impacts per day. Because of the small numbers of detected dust particles the statistical significance is relatively small so that new observations are required to confirm this result.

The event rate of the Pioneer 8 and 9 impacts reached a maximum peak just past the direction of the Sun. From this observation Whipple (1975) concluded that the direction of the impact center, which is strongly in the direction of the Sun, constrained that $\beta$-meteoroids on the whole are produced in orbits outside 0.5 AU. Whipple (1975) assumed a constant value for the angular momentum of a $\beta$-meteoroid that is released at zero velocity at perihelion. He varied the perihelion and aphelion distance to get information about the heliocentric distances at which the collisions occur. Even for an initial orbit that is parabolic, the minimum perihelion distance is $0.5 \mathrm{AU}$. This means that $\beta$-meteoroids are formed outside $0.5 \mathrm{AU}$ (Whipple 1975). Though both analyses refer to the defocusing period of the magnetic field, the result of Whipple (1975) does not contradict our conclusion that the place of origin of $\beta$-meteoroids is within a region of $0.5 \mathrm{AU}$ to the Sun. According to Hamilton et al. (1996) grains of radius $0.1 \mu \mathrm{m}$ remain bound for initial orbits between $0.75 \mathrm{AU}$ and 1.5 AU. Particles both interior and exterior to this disk escape from the ecliptic during the defocusing period. During the pole-to-pole passage of Ulysses the particles from the interior can be detected as $\beta$-meteoroids. The beginning of the Ulysses mission corresponds to the end of the focusing period of the magnetic field. At this time particles did not escape from the ecliptic according to Hamilton et al. (1996). Therefore, the place of origin of $\beta$-meteoroids can be determined to be within a region of $0.5 \mathrm{AU}$ to the Sun during the beginning of the Ulysses mission. 
The detection of $\beta$-meteoroids at high latitudes near the solar poles requires that the dust particles are deflected from their orbital motion within the ecliptic. Both interplanetary and interstellar particles suffer from this deflection which is caused only by the Lorentz force (Landgraf et al. 2000). Small interplanetary dust particles are ejected from a bigger particle which is moving in a bound orbit. On the other hand, small interstellar dust particles which are moving nearly perpendicularly to the Ulysses trajectory leave their initial orbit and reach the solar pole region due to the electromagnetic influence of the Lorentz force.

The detection of $\beta$-meteoroids within the northern hemisphere occurred for both orbits of Ulysses within a time range of nearly 4 months. During the first orbit $22 \beta$-meteoroids were identified in a distance range of 1.8 to $2.6 \mathrm{AU}$. Assuming an average speed of $30 \mathrm{~km} \mathrm{~s}^{-1}$ for these $\beta$-meteoroids their time of flight corresponds to a range of 3.7 to 5 months. Taking a time of nearly 4 months for the detection of these $22 \beta$-meteoroids into account, the time range in which these $\beta$-meteoroids were generated could be estimated to be 3 months. During the second orbit $16 \beta$-meteoroids were detected in a distance range of 1.3 to $1.9 \mathrm{AU}$. This corresponds to a time of flight between 2.5 and 3.7 months, i.e. $16 \beta$-meteoroids were generated in a time range of 4 months. From this we conclude that during the first orbit of Ulysses the production rate for $\beta$-meteoroids was nearly twice as high as during the second orbit. The identification of $\beta$-meteoroids that occurred during the first orbit of Ulysses at higher latitudes confirms this conclusion. Because the defocusing field was much more developed in 1995 during the first orbit than in 2002 during the second orbit, we can conclude that the defocusing magnetic field is responsible for this phenomenon. In 2002 the reversal of the polarity of the magnetic field caused the new focusing cycle to start. The difference in number of detected $\beta$-meteoroids between northern and southern hemisphere is remarkable. The quality of the observation of the dust detector, which can be characterized by the effective area, is similar for both hemispheres, as are the observing conditions relative to the magnetic cycle. So we cannot give any clear explanation for this asymmetry.

The high number of particles with velocities above $50 \mathrm{~km} \mathrm{~s}^{-1}$ in the upper right quadrant in Fig. 4 is notable. Obviously, it is a matter of particles on hyperbolic orbits far outside the solar region. During the time period from 16 August 1995 to 19 October 2001 we had a defocusing field. At this time the density of grains at higher latitudes increases as more grains are deflected away from the ecliptic into this region. In this context Hamilton et al. (1996) argued that the electromagnetic influence of the solar magnetic field, i.e. the Lorentz force $\boldsymbol{F}_{\mathrm{L}}$, is responsible for the motion of these dust particles, which are called electromagnetic $\beta$-meteoroids. At the end of the defocusing cycle in 2002 the regions of enhanced density move downstream and a focusing cycle begins to concentrate grains around the plane of the solar equator.

The flux of $\beta$-meteoroids was determined according to the following equation

$$
F(t)=\frac{1}{\Delta t} \int_{t}^{t+\Delta t} \frac{N\left(t^{\prime}\right)}{A_{\text {eff }}\left(t^{\prime}\right)} \mathrm{d} t^{\prime}
$$

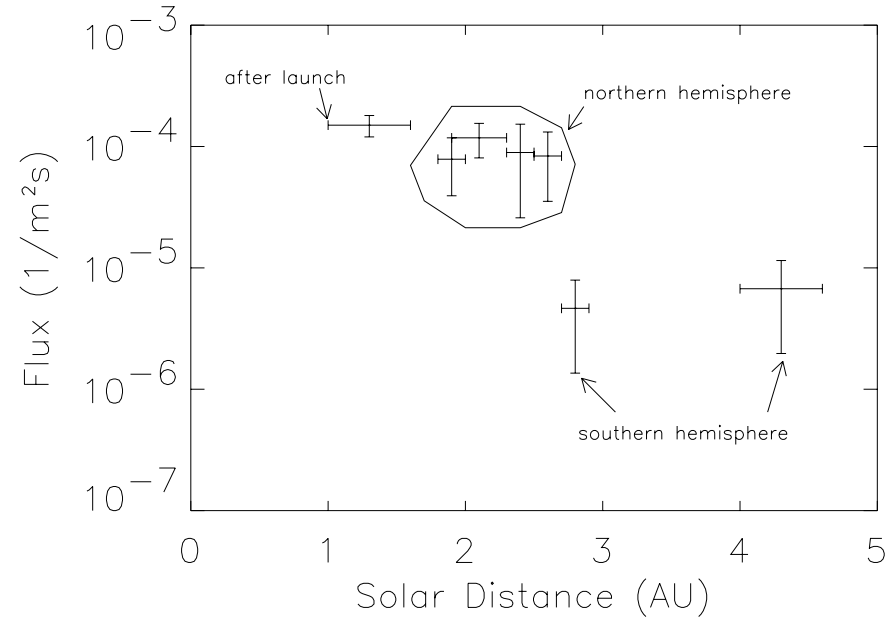

Fig. 6. The flux of $\beta$-meteoroids determined from the Ulysses data as a function of the solar distance. The flux of $1.5 \pm 0.3 \times 10^{-4} \mathrm{~m}^{-2} \mathrm{~s}^{-1}$ between 1.0 and $1.6 \mathrm{AU}$ in the ecliptic plane drops down to a value of $9.0 \pm 6.3 \times 10^{-5} \mathrm{~m}^{-2} \mathrm{~s}^{-1}$ between 1.8 and $2.7 \mathrm{AU}$ at solar ecliptic latitudes between $67^{\circ}$ and $79^{\circ}$ during the north polar passage. Within the southern hemisphere the flux equals $4.6 \times 10^{-6} \mathrm{~m}^{-2} \mathrm{~s}^{-1}$.

where $\Delta t$ represents the time interval of the measurement, $A$ is the effective area of the detector within this time interval and $N$ stands for the number of particles detected within the inter$\operatorname{val}\left[t^{\prime}, t^{\prime}+\mathrm{d} t^{\prime}\right]$.

As shown in Fig. 6 the flux of $\beta$-meteoroids during the first revolution around the Sun equals $1.5 \pm 0.3 \times 10^{-4} \mathrm{~m}^{-2} \mathrm{~s}^{-1}$ between 1.0 and $1.6 \mathrm{AU}$ in the ecliptic plane, and amounts to $9.0 \pm 6.3 \times 10^{-5} \mathrm{~m}^{-2} \mathrm{~s}^{-1}$ between 1.8 and $2.7 \mathrm{AU}$ at solar ecliptic latitudes between $67^{\circ}$ and $79^{\circ}$ during the north polar passage. Up to a solar distance of nearly 2.7 AU the decay of the flux of $\beta$-meteoroids with increasing solar distance can be approximated by a $r^{-\alpha}$-dependence with $\alpha \approx 2$. At greater solar distances probably the decreasing solar latitude leads to a lower flux of $\beta$-meteoroids. To estimate the flux for the second orbit of Ulysses we compare the effective area of the first with that of the second revolution. The flux of $\beta$-meteoroids is proportional to the number of $\beta$-meteoroids and inversely proportional to the effective sensor area. Comparing the maximum value of the effective area during the first revolution to the value during the second revolution of Ulysses within the northern hemisphere we find that they are of the same order of magnitude. Within the southern hemisphere the maximum value of the effective area during the second revolution of Ulysses reaches nearly $62.5 \%$ of the value during the first revolution. Because nearly the same number of $\beta$-meteoroids was detected during both orbits, we expect a similar flux of $\beta$-meteoroids for the second revolution of Ulysses.

\section{Summary}

The influence of the radiation pressure force and of the influence of the Lorentz force on the motion of dust particles has been investigated on the basis of the analysis of the Ulysses data. For the time period during the second revolution of Ulysses (16 August 1995-19 October 2001) the 
identification of $\beta$-meteoroids which are coming from a direction near to the Sun is based upon a criterion developed by Wehry \& Mann (1999). During this time period we identified 16 particles within the northern hemisphere as $\beta$-meteoroids coming from the Sun. An asymmetry between the detected $\beta$ meteoroids within the northern and the southern hemisphere could not be explained. The origin of the detected $\beta$-meteoroids has been determined to lie within a region of $0.5 \mathrm{AU}$ from the Sun. Because of different assumptions this result does not contradict to a study of Whipple (1975) who concluded that the origin was outside this region. The production rate of $\beta$ meteoroids during the first orbit of Ulysses has been estimated to be nearly twice of that during the second revolution. This is probably a result of the greater defocusing phase during the first orbit of Ulysses. Furthermore, we identified high-speed particles like the so-called electromagnetic $\beta$-meteoroids which do not come from the region near to the Sun. We estimated a similar flux of $\beta$-meteoroids for the second orbit of Ulysses. Until the end of 2008 we determined the expected effective area for particles which are coming on hyperbolic orbits from the direction of the Sun. This effective area lies high above the Solar poles.

\section{References}

Baguhl, M. 1993, Dissertation, Max-Planck-Institut für Kernphysik, Heidelberg

Berg, O. E., \& Grün, E. 1973, Space Res., 13, 1047

Grün, E., Fechtig, H., Giese, R. H., et al. 1992a, ApJS, 92, 411
Grün, E., Fechtig, H., Hanner, M. S., et al. 1992b, Space Sci. Rev. Ser., 60,317

Grün, E., Staubach, P., Baguhl, M., et al. 1997, Icarus, 129, 270

Grün, E., Baguhl, M., Svedhem, H., \& Zook, H. A. 2001, in Situ Measurements of Cosmic Dust in Interplanetary Dust, ed. E. Grün, B. A. S. Gustafson, S. Dermott, \& H. Fechtig (Berlin, Heidelberg, New York: Springer), 295

Hamilton, D. P., Grün, E., \& Baguhl, M. 1996, Electromagnetic escape of dust from the Solar System, in Physics, Chemistry, and Dynamics of Interplanetary Dust, ed. B. A. S. Gustafson, \& M. S. Hanner (Dordrecht: Kluwer), ASP Conf. Ser., 104, 31

Krüger, H., Grün, E., Landgraf, M., et al. 1999, Planet. Space Sci., 47, 363

Krüger, H., Grün, E., Landgraf, M., et al. 2001, Planet. Space Sci., 49, 1303

Landgraf, M., Augustsson, K., Grün, E., \& Gustafson, B. Å. S. 1999, Science, 286, 2319

Landgraf, M., Baggaley, W. J., Grün, E., Krüger, H., \& Linkert, G. 2000, Geophys. Res., 105(A5), 10343

Landgraf, M. 2000, Geophys. Res., 105(A5), 10303

Landgraf, M., Krüger, H., Altobelli, N., \& Grün, E. 2003, Geophys. Res., 108(A10), LIS 7-1

Parker, E. N. 1958, ApJ, 128, 664

Wehry, A., \& Mann, I. 1999, A\&A, 341, 296

Wehry, A. 2002, Dissertation, Max-Planck-Institut für Kernphysik, Ruprecht-Karls-Universität Heidelberg

Whipple, F. L. 1975, Sources of interplanetary dust, in Interplanetary Dust and Zodiacal Light, ed. H. Elsässer, \& H. Fechtig (New York: Springer-Verlag), Lect. Notes Phys., 48, 403

Zook, H. A., \& Berg, O. E. 1975, Planet. Space Sci., 23, 183 